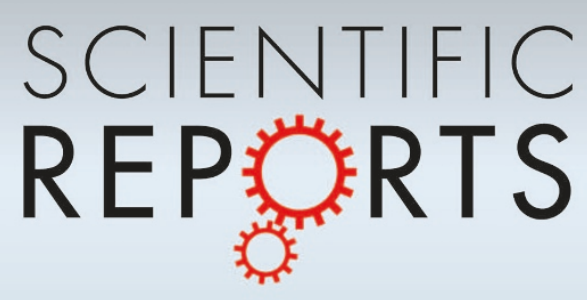

OPEN

SUBJECT AREAS: ELECTRONIC STRUCTURE

PHASE TRANSITIONS AND CRITICAL PHENOMENA

APPLIED MATHEMATICS

ELECTRONIC PROPERTIES AND MATERIALS

Received

26 June 2013

Accepted

27 August 2013

Published

18 September 2013

Correspondence and requests for materials should be addressed to

S.S. (s.sykora@ifwdresden.de)

\section{Heavy fermion properties of the Kondo Lattice model}

\author{
Steffen Sykora' \& Klaus W. Becker ${ }^{2}$
}

'IFW Dresden, P.O. Box 2701 16, 01171 Dresden, Germany, ${ }^{2}$ Institut für Theoretische Physik, Technische Universität Dresden, D01062 Dresden, Germany.

We study the $S=1 / 2$ Kondo lattice model which is widely used to describe heavy fermion behavior. In conventional treatments of the model the Kondo interaction is decoupled in favour of a hybridization of conduction and localized $f$ electrons. However, such an approximation breaks the local gauge symmetry and implicates that the local $f$-occupation is no longer conserved. To avoid these problems, we use in this work an alternative approach to the model based on the Projective Renormalization Method (PRM). Thereby, within the conduction electron spectral function we identify the lattice Kondo resonance as an almost flat excitation near the Fermi surface which is composed of conduction electron creation operators combined with localized spin fluctuations. This leads to an alternative description of the Kondo resonance without having to resort to an artificial symmetry breaking.

$\mathrm{n}$ the conventional view of heavy-fermion systems, conduction electrons ( $c$ electrons) and localized spins $(f$ electrons) hybridize with each other at low temperatures through the Kondo coupling and form a heavy Fermi liquid. Thereby, a large Fermi surface develops in this approach, which is determined by the total number of $c$ and $f$ electrons. This picture of heavy fermions is supported by experimental findings, since a narrow quasiparticle band is found in the excitation spectrum close to the Fermi energy ${ }^{1,2}$. From a theoretical point of view, a large Fermi surface follows within a factorization approximation of the Kondo coupling. In the Kondo lattice Hamiltonian

$$
\mathcal{H}=\sum_{\mathbf{k} \sigma} \varepsilon_{\mathbf{k}} c_{\mathbf{k} \sigma}^{\dagger} c_{\mathbf{k} \sigma}+j_{K} \sum_{i} \mathbf{S}_{i} \cdot \mathbf{s}_{i}=\mathcal{H}_{0}+\mathcal{H}_{1}
$$

$\mathcal{H}_{0}$ is the kinetic energy of the conduction electrons and $\mathcal{H}_{1}$ is the Kondo exchange $\left(j_{K}>0\right)$. $\mathrm{S}_{i}=\sum_{\alpha \beta}\left(\sigma_{\alpha \beta} / 2\right) f_{i \alpha}^{\dagger} f_{i \beta}$ and $\mathbf{s}_{i}=\sum_{\alpha \beta}\left(\boldsymbol{\sigma}_{\alpha \beta} / 2\right) c_{i \alpha}^{\dagger} c_{i \beta}$ represent the $f$ and $c$ electron spins. Combining an $f$ creation operator from $\mathrm{S}_{i}$ with a $c$ annihilation operator from $\mathrm{s}_{i}$, and vice versa, expectation values $\left\langle f_{i, \beta}^{\dagger} c_{i, \beta}\right\rangle$ and $\left\langle c_{i, \beta}^{\dagger} f_{i, \beta}\right\rangle$ can be formed, which lead to a mean-field model of hybridized $f$ and $c$ electrons. A more rigorous treatment of the Kondo lattice is based on the so-called large $N$ expansion. Here, $N$ is the $f$-spin degeneracy, which is artificially driven to infinity ${ }^{3,4}$. In the limit $N \rightarrow \infty$ the key physics is captured as a mean-field theory, and properties at finite $N$ are obtained through an expansion in the small parameter $1 / N$.

Due to the appearance of non-zero amplitudes $\left\langle f_{i, \beta}^{\dagger} c_{i, \beta}\right\rangle$ the mean-field description is related to a broken symmetry and a hybridization gap in the electronic spectrum. These artifacts are circumvented within our approach. A further outstanding drawback of the large $N$ approach is that the cross-over between the heavy Fermi liquid and the local moment physics appears as a sharp phase transition where the $1 / N$ expansion becomes singular ${ }^{5}$. Moreover, the large $N$ approach can not form two-particle singlets for $N>2$, such as Cooper pairs and spin-singlets. Therefore, anti-ferromagnetism and superconductivity are consequently absent from the meanfield theory ${ }^{5}$. Note that the local $f$-occupation, $n_{i}^{f}=\sum_{\sigma} f_{i \sigma}^{\dagger} f_{i \sigma}$, is a constant of motion of the Kondo lattice model, since $n_{i}^{f}$ commutes with the Kondo Hamiltonian. Therefore, the concept of a large Fermi surface in heavy fermions was modified by Oshikawa ${ }^{6}$ provided that the system can be described as a Fermi liquid. He showed, using rather general arguments that the Luttinger sum rule is fulfilled, when also the completely localized spins contribute to the Fermi sea volume as electrons.

However, it is not obvious that the size of the Fermi surface is large in the Kondo lattice model. In particular, in the one-dimensional case there is evidence based on Density Matrix Renormalization Group (DMRG) and Quantum Monte Carlo (QMC) calculations that the Fermi surface is small ${ }^{7-9}$. On the other hand, for an infinite-dimensional hypercubic lattice a Fermi-liquid state with a large Fermi surface was found ${ }^{10}$. In this study 
Dynamical Mean Field Theory (DMFT) and QMC was used avoiding breaking of the gauge symmetry. Evidence of a discontinuity was found in the momentum distribution indicating Fermi liquid behavior.

The DMFT is a widely-used approach to the Kondo lattice which relies on an expansion in powers of $1 / d$, where $d$ is a variable dimen$\operatorname{sion}^{11-13}$. The idea of the DMFT is to reduce the lattice problem to the physics of a single magnetic ion embedded within a self-consistently determined effective medium. The qualitative physics of the Kondo lattice, including the development of coherence at low temperatures, can well be described by this approach.

\section{Results}

Perturbation theory. The aim of the present work is to provide an alternative description of the transformation from localized moment to heavy Fermi liquid behavior as a smooth crossover instead of a sharp phase transition. To avoid technical details, at the beginning let us explain the main idea of our study on the basis of perturbation theory. These considerations will be used afterwards to construct an appropriate many-particle approach to numerically evaluate the electronic spectrum. The one-particle spectral function $A(\mathrm{k}, \omega)$ of conduction electrons is defined by $A(\mathrm{k}, \omega)=(1 / 2 \pi)$ $\int_{-\infty}^{\infty}\left\langle\left[c_{\mathrm{k} \sigma}(t), c_{\mathrm{k} \sigma}^{\dagger}\right]_{+}\right\rangle e^{i \omega t} d t$, where the expectation value and the time dependence are governed by Hamiltonian (1). The presence of the Kondo exchange prevents a straightforward evaluation of $A(\mathrm{k}, \omega)$. For that reason we transform the Hamiltonian into a diagonal (or at least quasi-diagonal) form by applying a unitary transformation to $\mathcal{H}, \tilde{\mathcal{H}}=e^{X} \mathcal{H} e^{-X}$. Here the generator $X=-X^{\dagger}$ in lowest order perturbation theory with respect to $j_{K}$ is given by

$$
X=\frac{1}{\sqrt{N_{L}}} \sum_{\mathrm{kk}^{\prime}} \frac{j_{K}}{\varepsilon_{\mathrm{k}}-\varepsilon_{\mathrm{k}^{\prime}}} \mathcal{K}_{\mathrm{kk}^{\prime}}
$$

( $N_{L}$ number of lattice sites). The operator quantity $\mathcal{K}_{\mathrm{kk}^{\prime}}=(1 / 2)$ $\sum_{\alpha \beta} \mathrm{S}_{\mathrm{k}^{\prime}-\mathrm{k}} \cdot \boldsymbol{\sigma}_{\alpha \beta} c_{\mathrm{k} \alpha}^{\dagger} c_{\mathrm{k}^{\prime} \beta}=\left(\mathcal{K}_{\mathrm{k}^{\prime} \mathrm{k}}\right)^{\dagger}$ is taken over from the decomposition of $\mathcal{H}_{1}=\left(j / \sqrt{N_{L}}\right) \sum_{\mathrm{kk}^{\prime}} \mathcal{K}_{\mathrm{kk}^{\prime}}$. Evaluating the transformation to second order in $j_{K}$, one finds

$$
\tilde{\mathcal{H}}=\sum_{\mathrm{k} \sigma} \tilde{\varepsilon}_{\mathrm{k}} c_{\mathrm{k} \sigma}^{\dagger} c_{\mathrm{k} \sigma}+\sum_{<i j>} \tilde{J}_{i j} \mathrm{~S}_{i} \cdot \mathrm{S}_{j}+\tilde{E},
$$

where $\tilde{\varepsilon}_{\mathrm{k}}$ is somewhat changed compared to $\varepsilon_{\mathrm{k}}$. The second term in (3) is the well-known RKKY interaction and $\tilde{E}$ is an additional energy constant. Note that in $\tilde{\mathcal{H}}$ the conduction electrons and the localized spins act as independent subsystems which no longer interact with each other. After an approximate diagonalization of the RKKY interaction, for instance by introducing Schwinger bosons, all expectation values formed with $\tilde{\mathcal{H}}$ can be determined. However, the evaluation of $A(\mathrm{k}, \omega)$ also requires the transformation of the operator from which the expectation value is taken. This follows from the general property $\langle\mathcal{A}\rangle=\left(\operatorname{Tr} \tilde{\mathcal{A}} e^{-\beta \tilde{\mathcal{H}}}\right) /\left(\operatorname{Tr} e^{-\beta \tilde{\mathcal{H}}}\right)=$ $\langle\tilde{\mathcal{A}}\rangle_{\tilde{\mathcal{H}}}$, for any operator variable $\mathcal{A}$, where $\tilde{\mathcal{A}}=e^{X} \mathcal{A} e^{-X}$. Thus, we obtain

$$
A(\mathrm{k}, \omega)=\frac{1}{2 \pi} \int_{-\infty}^{\infty}\left\langle\left[\tilde{c}_{\mathrm{k} \sigma}(t), \tilde{c}_{\mathrm{k} \sigma}^{\dagger}\right]_{+}\right\rangle_{\tilde{\mathcal{H}}} e^{i \omega t} d t
$$

where now the expectation value and the time dependence of $\tilde{c}_{\mathrm{k} \sigma}(t)$ are governed by $\tilde{\mathcal{H}}$. An explicit evaluation of $\tilde{c}_{\mathrm{k}, \sigma}^{\dagger}=e^{X} c_{\mathrm{k} \sigma}^{\dagger} e^{-X}$ up to second order in $X$ leads for $A(\mathrm{k}, \omega)$ to the expression

$A(\mathrm{k}, \omega)=\left|u_{\mathrm{k}}\right|^{2} \delta\left(\omega-\varepsilon_{\mathrm{k}}\right)+\frac{1}{4 N_{L}} \sum_{\mathrm{k}^{\prime}}\left|v_{\mathrm{k}^{\prime} \mathrm{k}}\right|^{2}\left\langle\mathrm{~S}_{\mathrm{k}-\mathrm{k}^{\prime}} \cdot \mathrm{S}_{\mathrm{k}^{\prime}-\mathrm{k}}\right\rangle_{\tilde{\mathcal{H}}_{\jmath}} \delta\left(\omega-\varepsilon_{\mathrm{k}^{\prime}}\right)$,

where the coefficients $u_{\mathrm{k}}$ and $v_{\mathrm{k}^{\prime} \mathrm{k}}$ depend on the initial dispersion of conduction electrons $\varepsilon_{\mathrm{k}}$ and the Kondo coupling $j_{K}$. Following
Doniach's picture of competing energy scales of anti-ferromagnetism and heavy fermion physics in $4 f$ systems $^{14,15}$, in Eq. (5) we have neglected the excitations of the localized spin system $\tilde{\mathcal{H}}_{J}=\sum_{\mathbf{q}} \tilde{J}_{\mathbf{q}} \mathrm{S}_{\mathbf{q}} \cdot \mathrm{S}_{-\mathbf{q}}$. Note that in the heavy-fermion regime the Kondo scale $T_{K}$ can be considered as large compared to the excitation energies of the $f$ system.

Thus, the one-particle spectrum (5) is built up by a coherent excitation with energy $\varepsilon_{\mathrm{k}}$ and amplitude $\left|u_{\mathrm{k}}\right|^{2}$ and by additional excitations at frequencies $\varepsilon_{\mathrm{k}^{\prime}}$ with amplitudes $\left|v_{\mathrm{k}^{\prime} \mathrm{k}}\right|^{2}$. Usually, such excitations are responsible for a typical broadening of the coherent excitation at $\varepsilon_{\mathrm{k}}$ and determine the lifetime of the quasiparticle in the metallic state. In the present case these are the contributions to $\left|v_{\mathrm{k}^{\prime} \mathrm{k}}\right|^{2}$ of order $j_{K}^{2}$. Beyond this second order perturbation theory we find that the next higher order $\propto j_{K}^{3}$ of the incoherent part of Eq. (5) leads to the well-known Kondo resonance which has been found by Kondo in case of one magnetic impurity. Namely, taking together in the second term the parts in $j_{K}^{2}$ and $j_{K}^{3}$ Eq. (5) can be rewritten as

$$
A(\mathrm{k}, \omega)=\delta\left(\omega-\varepsilon_{\mathrm{k}}\right)+\frac{1}{\pi} \frac{\Gamma(\omega)}{\left(\omega-\varepsilon_{\mathrm{k}}\right)^{2}}
$$

with

$\Gamma(\omega)=\frac{\pi S(S+1) j_{K}^{2}}{4 N_{L}} \sum_{\mathrm{k}^{\prime}}\left(1+\frac{2 j_{K}}{N_{L}} \sum_{\mathbf{q}} \frac{f\left(\varepsilon_{\mathbf{q}}\right)-\frac{1}{2}}{\varepsilon_{\mathrm{k}^{\prime}}-\varepsilon_{\mathbf{q}}}\right) \delta\left(\omega-\varepsilon_{\mathrm{k}^{\prime}}\right)+\cdots$,

where $\left|u_{\mathrm{k}}\right|^{2}$ was approximated by 1 . Note that in third order perturbation theory the expectation value $\left\langle\mathrm{S}_{\mathrm{k}-\mathrm{k}^{\prime}} \cdot \mathrm{S}_{\mathrm{k}^{\prime}-\mathrm{k}}\right\rangle_{\tilde{\mathcal{H}}_{J}} \approx S(S+1)$ becomes local, which means that all interaction effects between localized spins at different sites do not contribute since they are of higher order. Furthermore note that the sum over q leads to a divergence for $\varepsilon_{\mathrm{k}^{\prime}}=0$, and the additional excitations in $A(\mathrm{k}, \omega)$ become dominant. They are located close to the chemical potential $\omega=0$. Since their energy is momentum-independent, i.e. independent of $k$, this set of excitations has to be interpreted as the well-known Kondo resonance. The approximate result (7) agrees with the imaginary part of the Green's function for the impurity Kondo model in perturbation theory (compare e.g. with Ref. 16), where $A(\mathrm{k}, w)=(1 / \pi)$ $\Im G(\mathrm{k}, w)$. Using moreover the Green's function $G(\mathrm{k}, \omega)$ in the general form

$$
G(\mathrm{k}, \omega)=\frac{1}{\omega-\varepsilon_{\mathrm{k}}-i \Gamma(\omega)},
$$

(real part of the self energy is neglected) one also finds that $\Gamma(\omega)$ has to be interpreted as imaginary part of the one-particle self-energy. Thereby, higher order terms in the denominator of $A(\mathrm{k}, w)=(1 / \pi)$ $\Im G(\mathrm{k}, w)$ are neglected ${ }^{17}$.

Note that the perturbation expansion (7) of $\Gamma(\omega)$ in $j_{K}$ breaks down below a characteristic temperature when the second term in the bracket becomes of the order of the first term. This temperature is often used to define the Kondo temperature and is conventionally obtained from an expansion of an effective Kondo coupling ${ }^{18}$. To see this the sum over $\mathbf{q}$ can easily be evaluated

$\Gamma(\omega)=\frac{\pi S(S+1) j_{K}^{2}}{4 N_{L}} \sum_{\mathrm{k}^{\prime}}\left(1-2 \rho_{0} j_{K} \ln \frac{\max \left(\left|\varepsilon_{\mathrm{k}^{\prime}}\right|, T\right)}{D}\right) \delta\left(\omega-\varepsilon_{\mathrm{k}^{\prime}}\right)+\ldots$

or

$\Gamma(\omega)=\frac{\pi S(S+1) j_{K}^{2} \rho_{0}}{4}\left(1-2 \rho_{0} j_{K} \ln \frac{\max (|\omega|, T)}{D}\right)+\mathcal{O}\left(j_{K}^{4}\right)$,

where in the last line also the integration over $\mathrm{k}^{\prime}$ was performed. Here $\rho_{0}$ is the electronic density of states. In a final step the single-impurity Kondo temperature $T_{K}=D \exp \left(-1 / j_{K} \rho_{0}\right)$ is introduced leading to $\Gamma(\omega)=\frac{\pi S(S+1) j_{K}^{2} \rho_{0}}{4}\left(3-2 \rho_{0} j_{K} \ln \frac{\max (|\omega|, T)}{T_{K}}\right)+\mathcal{O}\left(j_{K}^{4}\right)$ 
One should again emphasize that no difference from the single impurity case $\mathrm{e}^{16,17}$ has occurred up to now.

The previous considerations are based on perturbation theory leading to a divergence in $A(\mathrm{k}, \omega)$. This divergence can be removed by including higher order contributions to infinite order in $j_{K}$. For the single impurity model, higher order perturbation theory leads to equally divergent contributions. This series was summed up by Abrikosov $^{19}$ in the leading $\ln \left(T / T_{K}\right)$ approximation to give the following result for the $T$-matrix, $T(\varepsilon) \simeq\left(j_{K} / N_{L}\right)\left(1-\left(j_{K} \rho_{0} / N\right.\right.$ $\ln (|\varepsilon| / D)))^{-1} s \cdot S$.

Non-perturbative many-particle approach to the $2 \mathrm{~d}$-Kondo lattice. The previous perturbation treatment leads to two characteristic problems in the evaluation of the one-particle spectral function $A(\mathrm{k}, \omega)$. (i) There are contributions to the spectral intensity which diverge and lead to unphysical results. (ii) There is no difference from the single impurity case in lowest order in $j_{K}$. These problems can only be solved by including higher order contributions to infinite order in $j_{K}$. In the following, we therefore apply the Projector-based Renormalization Method (PRM) ${ }^{20,21}$ to evaluate $A(\mathrm{k}, \omega)$ for the Kondo lattice. The general concept of the PRM (see methods section) is similar to what has been discussed above: To enable the solution of Hamiltonian (1), the Kondo interaction is again integrated out. However, a sequence of unitary transformations is used instead of a single transformation as in perturbation theory. Thereby, the PRM ensures a well-controlled disentanglement of higher order interaction terms leading to an effective Hamiltonian of the same operator structure as in Eq. (2). However, the renormalized parameters $\tilde{\varepsilon}_{\mathrm{k}}, \tilde{J}_{i j}$ and $\widetilde{E}$ are determined self-consistently taking into account contributions up to infinite order in the Kondo coupling. Moreover, within our nonperturbative approach expression (4) for $A(\mathrm{k}, \omega)$ is still valid. The amplitudes $u_{\mathrm{k}}$ and $v_{\mathrm{k}^{\prime}, \mathrm{k}}$ are also renormalized under the influence of higher order contributions and will be marked by tilde symbols as well. At this point we would like to emphasize the main motivation to develop our own theoretical treatment: Firstly, we note that the perturbative result (6) is also accessible within the PRM by expanding the renormalization equations up to lowest order. Furthermore, our approach has important advances over other theories for the Kondo lattice: The PRM neither suffers from

(a) $\mathrm{T}=0$

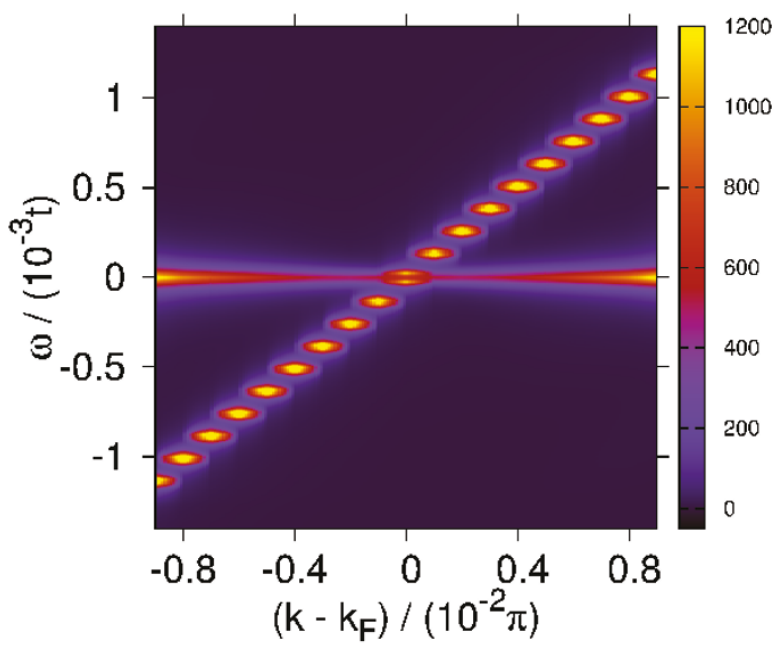

additional constraints, as in the large $N$ approach, nor from an expansion for large dimension $d$ as in the DMFT. Thereby, the local $f$-electron occupation $n_{i}^{f}$ remains a constant of motion in the calculation.

In the actual evaluation of the renormalization equations we restrict ourselves to two dimensions in order to minimize the numerical effort. Also, we consider electron concentrations only away from half-filling which is a special case since there the system becomes insulating. We have solved the renormalization equations self-consistently on a square lattice with $N_{L}=10^{3}$ lattice points. Fig. 1(a) shows $A(\mathrm{k}, \omega)$ for $T=0$ in a very small k-region around the Fermi momentum $k_{F}$ for electron filling $n_{c}=0.3$. Clearly one can see the coherent excitation $\tilde{\varepsilon}_{\mathrm{k}}$ which crosses the Fermi level at $\omega=0$. Note that the renormalization of $\tilde{\varepsilon}_{\mathrm{k}}$ is very weak. This behavior is found to be always true away from half-filling. In contrast, at half-filling $n_{c}=$ $1 / 2$ renormalization contributions to $\tilde{\varepsilon}_{\mathrm{k}}$ become strong due to nesting effects of the Fermi surface and lead to the opening of a gap at the Fermi energy. This results in an insulating phase $e^{22,23}$ and will be discussed in a forthcoming publication. Moreover, as expected, an additional almost k-independent excitation is found at the Fermi level which corresponds to additional contributions described by the second term in Eq. (5). In contrast to the perturbative treatment the renormalized amplitude $\tilde{v}_{\mathrm{k}^{\prime} \mathrm{k}}$ is no longer divergent in the PRM. However, it is still dominant for $\mathrm{k}^{\prime} \approx \mathrm{k}_{F}$ and any value of $\mathrm{k}$. As before, the multitude of these excitations has to be interpreted as Kondo resonance. It results from the second term in $A(\mathrm{k}, \omega)$ and not from a hybridization effect of $f$ and $c$ electrons. This important feature of the Kondo resonance can also be recognized in Fig. 1(a). In contrast to the coherent excitation, for the Kondo resonance we find a continuous spread of spectral intensity at the Fermi level throughout the whole momentum region. Due to the finite number of lattice points, considered in our calculation, the one-particle kinetic energy $\tilde{\varepsilon}_{\mathrm{k}}$ is defined only for a finite number of k-points. Therefore the coherent part of $A(\mathrm{k}, \omega)$, following the dispersion according to the first term of Eq. (5), shows a set of discrete bright spots (19 visible in Fig. 1) which is characteristic for a finite system. In contrast, the second part in Eq. (5) which contributes to the Kondo resonance is made up of an internal sum over all momenta $\mathrm{k}^{\prime}$ leading to the observed continuous momentum character. Considering finite electron systems, this characteristic feature could possibly be picked up by experiments to find

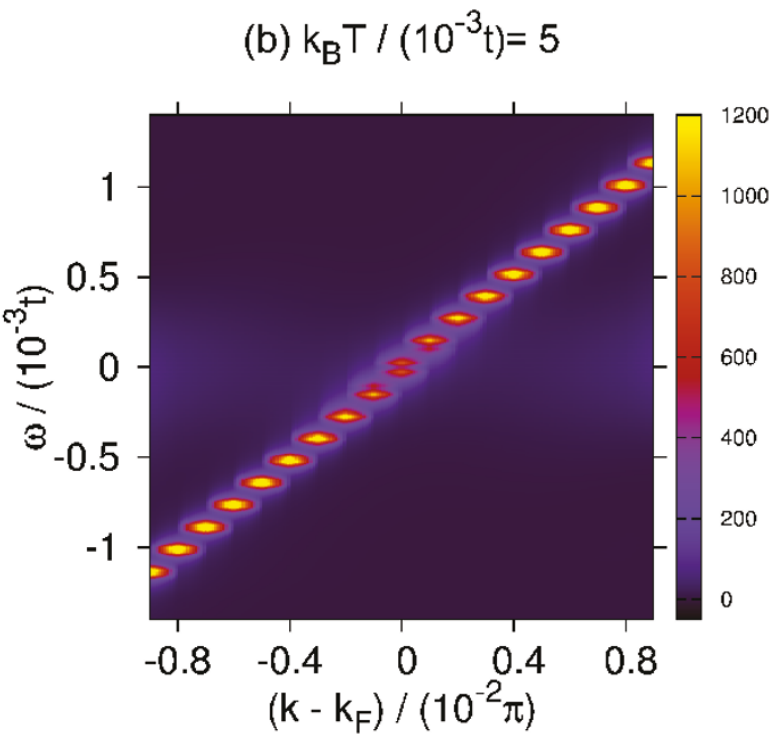

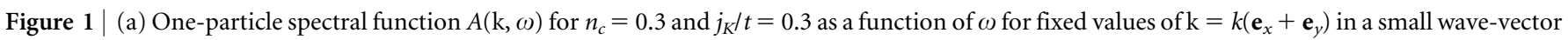

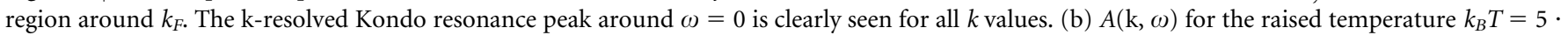

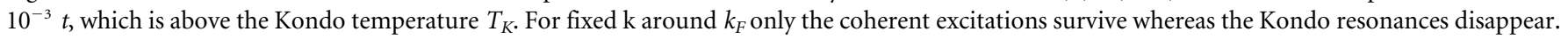
Note that the discrete bright spots for the coherent excitations are characteristic for finite systems which is not the case for the Kondo resonance. 
experimental evidence for the different nature of the Kondo resonance. In Fig. 1(b) the spectral function is given in the same wave-vector region as before for the raised temperature of $T=5$. $10^{-3} t$. Now the Kondo resonance has disappeared and only the coherent excitation has survived, which means that $T$ is higher than the Kondo temperature $T_{K}$. Let us mention that the overall behavior in Fig. 1 looks similar to numerical QMC results ${ }^{24,25}$ for the periodic Anderson model in one dimension for large values of the Coulomb repulsion $U$. As is well known, the Anderson model reduces to the Kondo model in the large $U$ limit and low lying energy level $\varepsilon_{f}$ of the localized $f$ electrons. In particular, in Ref. 24 some $f$-like bands with low weight were found very close to the chemical potential, two of which seemed almost not to disperse which is quite similar to the behavior of the resonance mode in Fig. 1.

Fig. 2 shows the density of states $\rho_{c}(\omega)=\left(1 / N_{L}\right) \Sigma_{\mathrm{k}} A(\mathrm{k}, \omega)$ as a function of $\omega$ for three different values of $T$ in a small frequency region around $\omega=0$. For the two lower $T$ the Kondo peak around $\omega$ $=0$ appears as a consequence of the Kondo contributions in $A(\mathrm{k}, \omega)$. The extra background in the spectrum is mainly composed of coherent excitations with respect to different momenta k. Additional incoherent excitations with energies different from zero may contribute to the background as well. We have also checked the sum rule $\int_{-\infty}^{\infty} A(\mathrm{k}, \omega) d \omega=1$, which at first glance seems to be contradicted by the obvious $T$-dependence of the different values in Fig. 2. However, the frequency interval outside the restricted $\omega$-region of Fig. 2 also contribute to the sum rule so that the sum rule is almost perfectly fulfilled for the different $T$ curves. Similarly, particle-hole symmetry seems to be present for the curves of Fig. 2. However this is not the case for $n_{c}=0.3$ when the full frequency region is considered (not shown).

\section{Discussion}

Firstly, let us discuss how the Kondo scale enters the formalism. To address this point, we start from relation $\sum_{i}\left\langle S_{i} \cdot S_{i}\right\rangle=\left(1 / \sqrt{N_{L}}\right)$ $\sum_{\mathrm{kk}^{\prime}}\left\langle\mathcal{K}_{\mathrm{k}^{\prime} \mathrm{k}}\right\rangle$, which suggests that the expectation value $\left\langle\mathcal{K}_{\mathrm{k}^{\prime} \mathrm{k}}\right\rangle$ participates in the formation of the singlet state at low temperatures. In Fig. 3 the expectation value $\left\langle\mathcal{K}_{\mathrm{k}^{\prime} \mathrm{k}}\right\rangle$ is shown for wave vectors close to the Fermi surface, $\mathrm{k}^{\prime}=\mathrm{k} \approx \mathrm{k}_{F}$. As expected, $\left\langle\mathcal{K}_{\mathrm{k}^{\prime} \mathrm{k}}\right\rangle$ turns out to be negative. Moreover, it exhibits a strong increase in magnitude with decreasing temperatures. This behavior is an indication for the

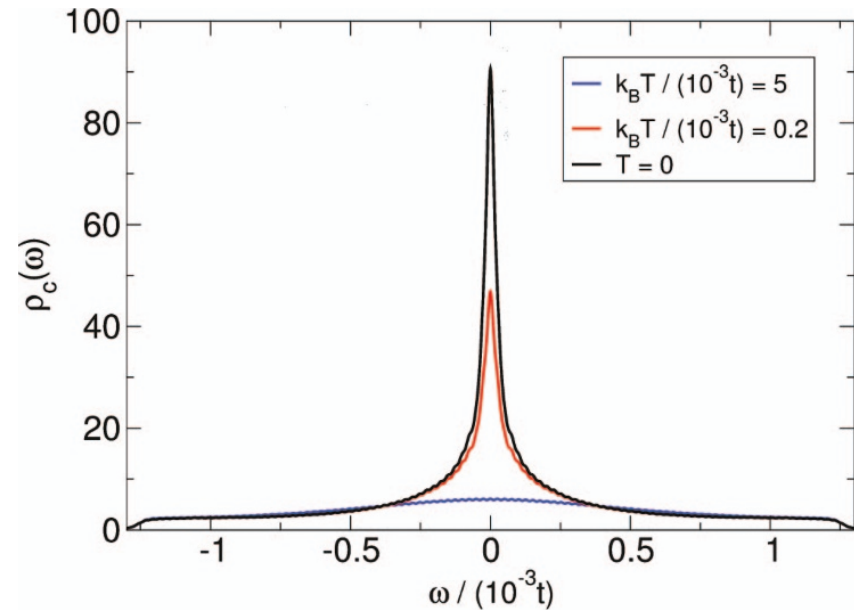

Figure $2 \mid$ Density of states $\rho_{c}(\omega)$ for $n_{c}=0.3$ and $j_{K} / t=0.3$ as a function of $\omega$ for three different temperatures, plotted for a small frequency region around $\omega=0$. Clearly seen is the Kondo resonance at $\omega=0$ for the two lower temperature values $T=0$ and $T=2 \cdot 10^{-4} t$. For $T=5 \cdot 10^{-3} t$ the resonance peak disappears since $T$ is larger than the Kondo temperature $T_{K} \approx 5 \cdot 10^{-4} t$ (see text). formation of the singlet state, to which predominantly wave vectors $\mathrm{k}^{\prime}, \mathrm{k}$ close to the Fermi surface participate. In real space this gives rise to correlations $\left\langle S_{i} \cdot s_{j}\right\rangle$ between local and conduction electron spins which are limited to a range of about 50 lattice sites (see inset). For comparison, Fig. 3 also shows the complete expectation value $\left\langle S_{i} \cdot s_{i}\right\rangle$ as a function of $T$. Obviously, $\left\langle\mathrm{S}_{i} \cdot \mathrm{s}_{i}\right\rangle$ is negative as well but almost independent of $T$. Because of the former relation between $\left\langle S_{i} \cdot s_{i}\right\rangle$ and $\left\langle\mathcal{K}_{\mathrm{k}^{\prime} \mathrm{k}}\right\rangle$ one concludes that expectation values $\left\langle\mathcal{K}_{\mathrm{k}^{\prime} \mathrm{k}}\right\rangle$ for wave vectors away from $k_{F}$ are not affected for low temperatures. These wave vectors form the majority in the Brillouin zone, which explains the marginal $T$-dependence of $\left\langle\mathrm{S}_{i} \cdot \mathrm{s}_{i}\right\rangle$. The width of $\left\langle\mathcal{K}_{\mathrm{kk}^{\prime}}\right\rangle$ in Fig. 3 can be identified with the Kondo scale $k_{B} T_{K}$ and is of order $5 \cdot 10^{-4} t$. The same energy scale is also found in the $T$-dependence of the electronic part of specific heat $C_{V}(T)$, which can be evaluated in the PRM as well (not shown). For low $T$ a strong increase $\sim T$ is found which is usually associated to a large heavy fermion mass. For $T>T_{K}$ the linear $T$-slope changes to a much lower value as known from normal metals.

In conclusion, we have applied the Projector-based Renormalization Method (PRM) to the Kondo lattice model in $d=2$ in order to study the electronic one-particle spectrum in the heavy-fermion regime. As main result of our study, we have found that the Kondo resonance is caused by additional low-energetic contributions to the spectral function $A(\mathrm{k}, \omega)$, which are dispersionless and distributed continuously in momentum space even for a finite electron system. Such a continuous distribution disagrees with a mean-field description of the Kondo resonance and proves that the Kondo resonance is a true many-body effect.

Looking at the electron density $n_{c}$, one finds that $n_{c}$ is related to the electronic spectral function via $n_{c}=\left(1 / N_{L}\right) \sum_{\mathrm{k}} \int A^{-}(\mathrm{k}, \omega) d \omega$. On the right hand side, all excitations from the non-perturbative extension of Eq. (5) contribute. This relation fixes the position of the Fermi energy, when $n_{c}$ is fixed. Using our result for $A(\mathrm{k}, \omega)$, the Kondo-type excitation turns out to be located slightly below the Fermi energy, i.e. inside the Fermi volume, which is consistent with photoemission experiments. This low-energy quasi-particle is the famous Abrikosov-Suhl resonance indicating heavy fermion behavior which is mainly characterized by a large effective mass of conduction electrons. The resonance emerges from the second term in Eq. (5) in

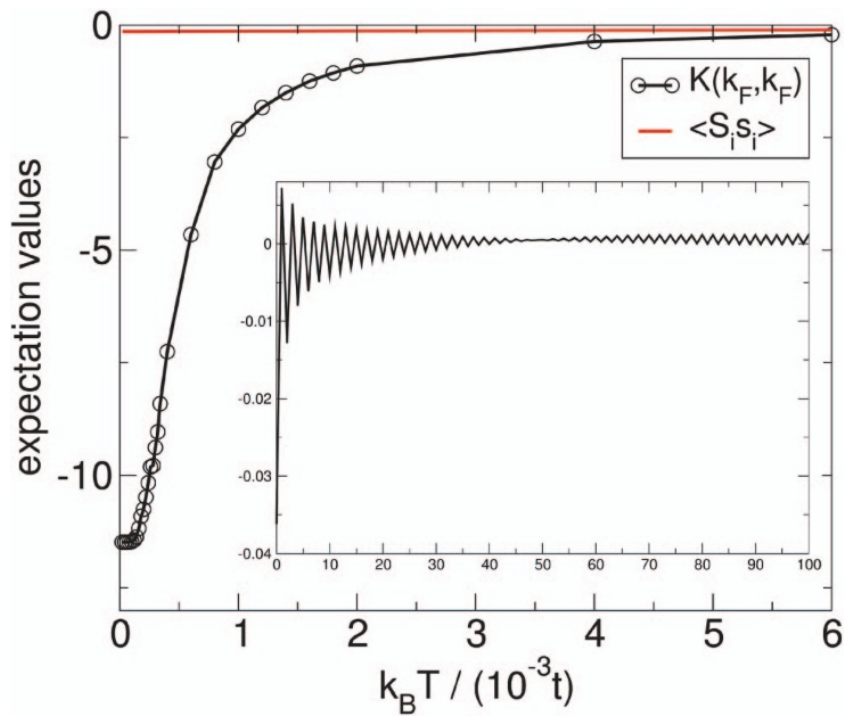

Figure $3 \mid$ Spin coupling $\left\langle\mathcal{K}_{\mathbf{k}^{\prime} \mathbf{k}}\right\rangle$ for $\boldsymbol{k}^{\prime}=k \approx k_{F}$ as a function of $T$ (black curve). For $T$ below $T_{K}\left\langle\mathcal{K}_{\mathrm{k}^{\prime} \mathrm{k}}\right\rangle$ drops to rather small values which indicates the formation of the heavy-fermion state. In contrast, $\left\langle S_{i} \cdot s_{i}\right\rangle$ is almost $T$ independent (red curve). This behavior demonstrates that only wave vectors close to $k_{F}$ contribute to the formation of the heavy-fermion state. The inset shows the correlation function $\left\langle S_{i} \cdot s_{j}\right\rangle$ as function of $(i-j)$. 
addition to the conventional coherent contribution. Thus, experimentalists might interpret both excitation types, i.e. the coherent excitation $\tilde{\varepsilon}_{\mathrm{k}}$ and the Kondo resonance, as ingredients of the large Fermi volume within a Fermi liquid picture resulting from a meanfield description.

Unfortunately, the question whether the Kondo lattice in $2 d$ is a Fermi liquid can not be answered by our present treatment. On the one hand, for the electron filling $n_{c}=0.3$ considered in this work the results are comparable with the Kondo impurity model, which is known to be a Fermi liquid ${ }^{19,26,27}$. The Kondo resonance always arises at $\varepsilon_{\mathrm{k}} \approx 0$, i.e. at $\omega \approx 0$ and is wave-vector independent as in the single impurity case. Moreover, we have found that the wave-vector dependence of the spin correlation function $\left\langle S_{\mathrm{k}-\mathrm{k}^{\prime}} \cdot \mathrm{S}_{\mathrm{k}^{\prime}-\mathrm{k}}\right\rangle$ is rather smooth, i.e. the correlations between local spins at different sites are rather weak. Therefore, we conclude that for parameter values not too close to the quantum phase transition to antiferromagnetism, the impurity physics largely governs the Kondo physics of the lattice model at $n_{c}=0.3$. On the other hand, one should emphasize that correlation effects between different lattice sites were found in the spin correlation function $\left\langle S_{i} \cdot s_{j}\right\rangle$ which is spatially extended in a range of 50 lattice sites (inset of Fig. 3). Thus, long ranging coherence effects between local and conduction electron spins do not disturb the impurity-like behavior of the Kondo lattice. Moreover, for $\mathrm{k}$ values on the Fermi surface, $\mathrm{k}=\mathrm{k}_{\mathrm{F}}$, the numerical evaluation of the quasiparticle weight $\left|\tilde{u}_{\mathrm{k}}\right|^{2}$ leads to a very small value. A nonvanishing of the quasiparticle weight $\left|\tilde{u}_{\mathrm{k}}\right|^{2}$ (usually called $Z_{\mathrm{k}}$ ) would be an important evidence that the Kondo lattice in $d=2$ has the character of a Fermi liquid.

A possibly simple way to check experimentally the Fermi liquid behavior is to measure the k-dependent occupation number. Note that at finite temperatures the expected jump at $\mathrm{k}_{\mathrm{F}}$ for $T=0$ is smeared out. Therefore, one has to extract the finite temperature effects by use of an adequate Fermi distribution function, which is the usual way to interpret photoemission experiments at low temperatures. A jump at $\mathrm{k}_{F}$ would support the Fermi liquid picture.

\section{Methods}

Projector-based renormalization method (PRM). The PRM starts from a decomposition of a many-particle Hamiltonian $\mathcal{H}$ into an unperturbed part $\mathcal{H}_{0}$ and into a perturbation $\mathcal{H}_{1}$. The latter part accounts for transitions between the eigenstates of $\mathcal{H}_{0}$. The basic idea of the PRM is to integrate out the perturbation $\mathcal{H}_{1}$ by a series of unitary transformations. For practical applications the unitary transformations are best done in small energy steps $\Delta \lambda$. Thereby, the evaluation in each step can be restricted to low orders in $\mathcal{H}_{1}$. This procedure usually limits the validity of the approach to parameter values of $\mathcal{H}_{1}$ which are of the same magnitude as those of $\mathcal{H}_{0}$. Assuming that all transitions with energies larger than some energy cutoff $\lambda$ are already integrated out, the transformed Hamiltonian $\mathcal{H}_{\lambda}$ for the Kondo lattice model reads, $\mathcal{H}_{\lambda}=\mathcal{H}_{0, \lambda}+\mathcal{H}_{1, \lambda}$ with

$$
\begin{gathered}
\mathcal{H}_{0, \lambda}=\sum_{\mathbf{k} \sigma} \varepsilon_{\mathbf{k}, \lambda} c_{\mathbf{k} \sigma}^{\dagger} c_{\mathbf{k} \sigma}+\sum_{\mathbf{q}} J_{\mathbf{q}, \lambda} S_{\mathbf{q}} \cdot S_{-\mathbf{q}}+E_{\lambda} \\
\mathcal{H}_{1, \lambda}=\frac{1}{\sqrt{N_{L}}} \sum_{\mathbf{k} \mathbf{k}^{\prime}} j_{\mathbf{k}^{\prime} \mathbf{k}, \lambda} \Theta\left(\lambda-\left|\varepsilon_{\mathbf{k}^{\prime}, \lambda}-\varepsilon_{\mathbf{k}, \lambda}\right|\right) \mathcal{K}_{\mathbf{k}^{\prime} \mathbf{k}},
\end{gathered}
$$

where the operator quantity $\mathcal{K}_{\mathbf{k}^{\prime} \mathbf{k}}$ was introduced before. Due to the elimination of high-energy transitions above $\lambda$ the coefficients in Eqs. (12),(13) depend on $\lambda$. As in the perturbative treatment, an effective exchange interaction between the $f$ spins as well as an energy constant $E_{\lambda}$ and a new k-dependence of the Kondo coupling $j_{K}$ are generated by the transformation. The $\Theta$-function in $\mathcal{H}_{1, \lambda}$ limits the excitations to energies smaller than $\lambda$. To find the $\lambda$-dependence of the coefficients in $\mathcal{H}_{\lambda}$ we study a subsequent unitary transformation of $\mathcal{H}_{\lambda}$ to a new Hamiltonian $\mathcal{H}_{\lambda-\Delta \lambda}$

$$
\mathcal{H}_{\lambda-\Delta \lambda}=e^{X_{\lambda, \Delta \lambda}} \mathcal{H}_{\lambda} e^{-X_{\lambda, \Delta \lambda}}
$$

Thereby, all transitions between $\lambda$ and a somewhat reduced cutoff $\lambda-\Delta \lambda$ will be eliminated. The generator $X_{\lambda, \Delta \lambda}$ of the unitary transformation

$$
\begin{aligned}
& X_{\lambda, \Delta \lambda}=\frac{1}{\sqrt{N_{L}}} \sum_{\mathbf{k k}^{\prime}} X_{\mathbf{k}^{\prime} \mathbf{k}}(\lambda, \Delta \lambda) \mathcal{K}_{\mathbf{k}^{\prime} \mathbf{k}} \\
& X_{\mathbf{k}^{\prime} \mathbf{k}}(\lambda, \Delta \lambda)=\frac{j_{\mathbf{k}^{\prime} \mathbf{k}, \lambda}}{\varepsilon_{\mathbf{k}^{\prime}, \lambda}-\varepsilon_{\mathbf{k}, \lambda}} \Theta_{\mathbf{k}^{\prime} \mathbf{k}, \lambda}\left(1-\Theta_{\mathbf{k}^{\prime} \mathbf{k}, \lambda-\Delta \lambda}\right),
\end{aligned}
$$

$\left(\Theta_{\mathbf{k}^{\prime} \mathbf{k}, \lambda}=\Theta\left(\lambda-\left|\varepsilon_{\mathbf{k}^{\prime}, \lambda}-\varepsilon_{\mathbf{k}, \lambda}\right|\right)\right.$, is fixed by the requirement that $\mathcal{H}_{\lambda-\Delta \lambda}$ no longer contains excitations with energies larger than $\lambda-\Delta \lambda$. Since the elimination of transitions is confined to a small energy shell $\Delta \lambda$, the evaluation of $\mathcal{H}_{\lambda-\Delta \lambda}$ from $\mathcal{H}$ can be restricted to low order contributions in $j_{\mathbf{k}^{\prime} \mathbf{k}, \lambda}$. Expression (15) is a slight generalization of the generator $X$, which was used to derive $\tilde{\mathcal{H}}$ in Eq. (2). By evaluating transformation (14) to order $j_{\mathbf{k k}^{\prime}, \lambda}^{2}$ together with (12), (13) we obtain discrete renormalization equations (or flow equations) connecting the coefficients at cutoff $\lambda$ with those at cutoff $\lambda-\Delta \lambda$. For instance, the renormalization equation for $j_{\mathbf{k}^{\prime} \mathbf{k}, \lambda}$ reads

$$
j_{\mathbf{k}^{\prime} \mathbf{k}, \lambda-\Delta \lambda}-j_{\mathbf{k}^{\prime} \mathbf{k}, \lambda}=\frac{1}{2 N_{L}} \sum_{\mathbf{q}} \Gamma_{\mathbf{k} \mathbf{q} ; \mathbf{k}^{\prime} \mathbf{q}}^{\lambda}\left(2\left\langle c_{\mathbf{q} \sigma}^{\dagger} c_{\mathbf{q} \sigma}\right\rangle\right)
$$

where $\Gamma_{\mathbf{q}^{\prime} \mathbf{q} ; \mathbf{k}^{\prime} \mathbf{k}}^{\lambda}=\Theta_{\mathbf{q q}^{\prime}, \lambda} j_{\mathbf{q q}^{\prime} \lambda} X_{\mathbf{k}^{\prime} \mathbf{k}}(\lambda, \Delta \lambda)+\Theta_{\mathbf{k k}^{\prime}, \lambda} j_{\mathbf{k k}^{\prime}, \lambda} X_{\mathbf{q}^{\prime} \mathbf{q}}(\lambda, \Delta \lambda)$. The complete elimination procedure starts from the original model (1), where the coefficients are fixed to $\varepsilon_{\mathbf{k}, \Lambda}=\varepsilon_{\mathbf{k}}, J_{\mathbf{q}, \Lambda}=0, E_{\Lambda}=0, j_{\mathbf{k k}^{\prime}, \Lambda}=j_{K}$. Here $\lambda=\Lambda$ denotes the largest transition energy of the original Hamiltonian (1). Proceeding in steps $\Delta \lambda$ until $\lambda=0$ is reached all transition operators from $\mathcal{H}_{1}$ will be used up. One arrives at the final Hamiltonian $\mathcal{H}_{\lambda=0}$, which has exactly the same form as the Hamiltonian $\tilde{\mathcal{H}}$ of Eq. (3). Thus, from now on quantities with tilde symbols will always denote the fully renormalized quantities at cutoff $\lambda=0$. Note that the explicit evaluation of the unitary transformation leads at first to operator expressions which differ from those of the generic ansatz (12), (13). Therefore, an additional factorization approximation has to be performed in order to trace back these operators to the generic ones. As a result, the renormalization equations also depend on expectation values $\langle\cdots\rangle$, which have to be solved self-consistently together with the renormalization equations of $\mathcal{H}_{\lambda}$. For the evaluation of these expectation values we use the invariance property $\langle\mathcal{A}\rangle=\langle\tilde{\mathcal{A}}\rangle_{\tilde{H}}$ To find the desired spectral function $A(\mathbf{k}, \omega)$ we start from Eq. (4) with $\tilde{c}_{\mathbf{k}, \sigma}^{\dagger}=c_{\mathbf{k} \sigma}^{\dagger}(\lambda=0)$. The $\lambda$-dependence of $c_{\mathbf{k} \sigma}^{\dagger}(\lambda)$ is calculated via the following ansatz:

$$
c_{\mathbf{k} \sigma}^{\dagger}(\lambda)=u_{\mathbf{k}, \lambda} c_{\mathbf{k} \sigma}^{\dagger}+\frac{1}{\sqrt{N}_{L}} \sum_{\mathbf{k}^{\prime}} v_{\mathbf{k}^{\prime}, \mathbf{k}, \lambda} \sum_{\alpha} \mathbf{S}_{\mathbf{k}-\mathbf{k}^{\prime}} \frac{\boldsymbol{\sigma}_{\alpha \sigma}}{2} c_{\mathbf{k}^{\prime} \alpha}^{\dagger}
$$

with initial conditions $u_{\mathbf{k}, \Lambda}=1, v_{\mathbf{k}^{\prime} \mathbf{k}, \Lambda}=0$. The operator structure of (17) is suggested from the first order expansion in $X_{\lambda, \Delta \lambda}$. The renormalization equations for the $\lambda$ dependent coefficients $u_{\mathbf{k}, \lambda}$ and $v_{\mathbf{k}^{\prime} \mathbf{k}, \lambda}$ are obtained by transforming $c_{\mathbf{k} \sigma}^{\dagger}(\lambda)$ to $c_{\mathbf{k} \sigma}^{\dagger}(\lambda-\Delta \lambda)$ in analogy to the transformation of $\mathcal{H}_{\lambda}$. The equation for $v_{\mathbf{k}, \mathbf{k}^{\prime}, \lambda}$ to first order in $X_{\lambda, \Delta \lambda}$ reads

$v_{\mathbf{k}^{\prime}, \mathbf{k}, \lambda-\Delta \lambda}-v_{\mathbf{k}^{\prime}, \mathbf{k}, \lambda}=u_{\mathbf{k}, \lambda} X_{\mathbf{k}^{\prime} \mathbf{k}}(\lambda, \Delta \lambda)+\frac{1}{2 N_{L}} \sum_{\mathbf{q}} v_{\mathbf{q} \mathbf{k}, \lambda} X_{\mathbf{k}^{\prime} \mathbf{q}}(\lambda, \Delta \lambda)\left(2\left\langle c_{\mathbf{q} \alpha}^{\dagger} c_{\mathbf{q} \alpha}\right\rangle-1\right)$,

The renormalization equation for $u_{\mathbf{k}, \lambda}$ can be evaluated in the same way. Alternatively, it can be found from the sum rule $\left\langle\left[c_{\mathbf{k}, \sigma}(\lambda), c_{\mathbf{k}, \sigma}^{\dagger}(\lambda)\right]_{+}\right\rangle=1$, with $\lambda$ replaced by $\lambda-$ $\Delta \lambda$

$$
\begin{aligned}
\left|u_{\mathbf{k}, \lambda-\Delta \lambda}\right|^{2}= & 1-\frac{1}{4 N_{L}} \sum_{\mathbf{q}}\left|v_{\mathbf{q} \mathbf{k}, \lambda-\Delta \lambda}\right|^{2}\left\langle\mathrm{~S}_{\mathbf{k}-\mathbf{q}} \cdot \mathrm{S}_{\mathbf{q}-\mathbf{k}}\right\rangle- \\
& \frac{1}{2 N_{L}^{3 / 2}} \sum_{\mathbf{q \mathbf { q } ^ { \prime }}} v_{\mathbf{q} \mathbf{k}, \lambda-\Delta \lambda} v_{\mathbf{q}^{\prime} \mathbf{k}, \lambda-\Delta \lambda}^{*}\left\langle\mathcal{K}_{\mathbf{q \mathbf { q } ^ { \prime }}}\right\rangle .
\end{aligned}
$$

Note that due to (18) the coefficients $v_{\mathbf{q}, \mathbf{k}, \lambda-\Delta \lambda}$ and $v_{\mathbf{q}^{\prime}, \mathbf{k}, \lambda-\Delta \lambda}$ in (19) at cutoff $\lambda-\Delta \lambda$ can be expressed by $v_{\mathbf{k}^{\prime}, \mathbf{k}, \lambda}$ and $u_{\mathbf{k}, \lambda}$. Thus, equations (18), (19) relate the two quantities $u_{\mathbf{k}, \lambda-\Delta \lambda}$ and $v_{\mathbf{k}^{\prime}, \mathbf{k}, \lambda-\Delta \lambda}$ at cutoff $\lambda-\Delta \lambda$ with the same quantities at $\lambda$. Since the renormalized Hamiltonian $\mathcal{H}_{\lambda=0}=\mathcal{H}$ has the same decoupled form (3) as in perturbation theory, the renormalization equations (18), (19) together with (17) lead to the final result for the spectral function $A(\mathbf{k}, \omega)$. Thereby, $A(\mathbf{k}, \omega)$ takes the same form as (5) but with the fully renormalized parameters of Hamiltonian $\tilde{\mathcal{H}}$ and of $\tilde{c}_{\mathbf{k} \sigma}^{\dagger}$.

As mentioned above, the Kondo resonance results from additional contributions to the spectral function $A(\mathbf{k}, \omega)$ close to the Fermi surface. Looking at expression (5) for $A(\mathbf{k}, \omega)$ one notices that only terms from the sum over $\mathbf{k}^{\prime}$ can contribute to the Kondo resonance which have $\mathbf{k}^{\prime} \approx \mathbf{k}_{F}$, so that $\varepsilon_{\mathbf{k}^{\prime}} \approx \varepsilon_{\mathbf{k}_{\mathrm{F}}}=0$. At the same time, also the prefactors $\left|\tilde{v}_{\mathbf{k}^{\prime}, \mathbf{k}}\right|^{2}$ become large for the same $\mathbf{k}^{\prime}$ values. This latter property can be understood by means of renormalization equation (17) for $v_{\mathbf{k}^{\prime} \mathbf{k}, \lambda}$ : Taking $\mathbf{k}^{\prime} \approx \mathbf{k}_{F}$ and arbitrary values of $\mathbf{k} \neq \mathbf{k}_{F}$, the first term $u_{\mathbf{k}, \lambda} X_{\mathbf{k}^{\prime} \mathbf{k}}(\lambda, \Delta \lambda)$ on the right hand side of (18) is nonzero but small since $X_{\mathbf{k}^{\prime} \mathbf{k}}(\lambda, \Delta \lambda)$ is small. This follows from the structure of $X_{\mathbf{k}^{\prime} \mathbf{k}}(\lambda$, $\Delta \lambda)$ in (15). Similarly, in the second term of Eq. (18) those terms from the sum over $q$ contribute most for which $\mathbf{q} \approx \mathbf{k}_{F}$, since then $X_{\mathbf{k}^{\prime} \mathbf{q}}(\lambda, \Delta \lambda)$ is large. Thus, one concludes that terms with wave vectors $\mathbf{k}^{\prime} \approx \mathbf{k}_{F}$ lead to a finite value of $v_{\mathbf{k}^{\prime} \mathbf{k}, \lambda}$ in the renormalization procedure for $\lambda \rightarrow 0$. This property explains the appearance of the Kondo resonance in $A(\mathbf{k}, \omega)$, which is almost independent of $\mathbf{k}$ [Fig. 1]. On the other hand, from sum rule (19) one can show that for $\lambda \rightarrow 0$ the renormalized amplitude $\left|\tilde{u}_{\mathbf{k}}\right|^{2}$ of the coherent excitation in $A(\mathbf{k}, \omega)$ becomes reduced. For the case $\mathbf{k}=\mathbf{k}_{F}$, which was excluded above, the numerical evaluation of the renormalization equations (18), (19) leads to a very small value of the coherent coefficient $\left|\tilde{u}_{\mathbf{k}}\right|^{2}$, which can not be distinguished from zero within the numerical accuracy.

1. Andres, K., Graebner, J. E. \& Ott, H. R. $4 f$-virtual-bound-state formation in $\mathrm{CeAl}_{3}$ at low temperatures. Phys. Rev. Lett. 35, 1779-1782 (1975).

2. Grewe, N. \& Steglich, F. Handbook On The Physics And Chemistry Of Rare Earths [343] (North-Holland 39 1991). 
3. Coleman, P. 1/N expansion for the Kondo lattice. Phys. Rev. B 28, 5255-5262 (1983).

4. Read, N., Newns, D. M. \& Doniach, S. Stability of the Kondo lattice in the large-N limit. Phys. Rev. B 30, 3841-3844 (1984).

5. Coleman, P. Introduction To Many Body Physics (Cambridge University Press, in press).

6. Oshikawa, M. Commensurability, excitation gap, and topology in quantum many-particle systems on a periodic lattice. Phys. Rev. Lett. 84, 1535-1538 (2000).

7. Troyer, M. \& Würtz, D. Ferromagnetism of the one-dimensional Kondo-lattice model: a quantum Monte Carlo study. Phys. Rev. B 47, 2886-2889 (1993).

8. Xavier, J. C., Novais, E. \& Miranda, E. Small Fermi surface in the one-dimensional Kondo lattice model. Phys. Rev. B 65, 214406 (2002).

9. Basylko, S. A., Lundow, P. H. \& Rosengren, A. One-dimensional Kondo lattice model studied through numerical diagonalization. Phys. Rev. B 77, 073103 (2008).

10. Otsuki, J., Kusunose, H. \& Kuramoto, Y. Evolution of a large Fermi surface in the Kondo lattice. Phys. Rev. Lett. 102, 017202 (2009).

11. Metzner, W. \& Vollhardt, D. Correlated lattice fermions in $d=\infty$ dimensions. Phys. Rev. Lett. 62, 324-327 (1989)

12. Georges, A., Kotliar, G., Krauth, W. \& Rozenberg, M. J. Dynamical mean-field theory of strongly correlated fermion systems and the limit of infinite dimensions. Rev. Mod. Phys. 68, 13-125 (1996)

13. Jarrell, M. Hubbard model in infinite dimensions: a quantum Monte Carlo study. Phys. Rev. Lett. 69, 168-171 (1992).

14. Doniach, S. The Kondo lattice and weak antiferromagnetism. Physica B 91, 231-234 (1977).

15. Kondo, J. Resistance minimum and heavy fermions. Proc. Jpn. Acad., Ser. B 82, 328-338 (2006).

16. Nagaoka, Y. Self-consistent treatment of low-temperature anomalies due to the $s$ $d$ exchange interaction. Prog. Theor. Phys. 37, 13-28 (1967).

17. Nagaoka, Y. Self-consistent treatment of Kondo's effect in dilute alloys. Phys. Rev. 138, A1112-A1120 (1965)

18. Fulde, P. Electron Correlations In Molecules And Solids (Springer Series in SolidState Sciences 100, Springer-Verlag, Berlin, 1991).

19. Yamada, K. Electron Correlations In Metals (Cambridge University Press, 2004).

20. Becker, K. W., Hübsch, A. \& Sommer, T. Renormalization approach to manyparticle systems. Phys. Rev. B 66, 235115 (2002).
21. Sykora, S., Becker, K. W. \& Fehske, H. Charge-density-wave formation in a halffilled fermionboson transport model: a projective renormalization approach. Rev Rev. B 81, 195127 (2010).

22. Watanabe, S., Kuramoto, Y., Nishino, T. \& Shibata, N. Spin, charge and quasiparticle gaps in the one-dimensional Kondo lattice with $f^{2}$ configuration. J. Phys. Soc. Jpn. 68, 159-165 (1999).

23. Assaad, F. F. Quantum Monte Carlo simulations of the half-filled twodimensional Kondo lattice model. Phys. Rev. Lett. 83, 796-799 (1999).

24. Gröber, C. \& Eder, R. Fermiology of a one-dimensional heavy-electron metal. Phys. Rev. B 59, R10405-R10408 (1999).

25. Tsutsui, K. et al. Heavy quasiparticles in the Anderson lattice model. Phys. Rev. Lett. 76, 279-282 (1996).

26. Hewson, A. C. The Kondo Problem To Heavy Fermions (Cambridge University Press, 1993).

27. Takano, F. \& Ogawa, T. Simple self-consistent treatment of Kondo's effect in dilute alloys. Prog. Theor. Physics. 35, 343-356 (1966).

\section{Acknowledgements}

We would like to thank J. van den Brink for helpful discussions.

\section{Author contributions}

K.W.B. initiated the project. S.S. and K.W.B. developed the theory. S.S. performed the numerical calculations. S.S. and K.W.B. wrote the main manuscript text and S.S. prepared the figures. All authors discussed the results and reviewed the manuscript.

\section{Additional information}

Competing financial interests: The authors declare no competing financial interests.

How to cite this article: Sykora, S. \& Becker, K.W. Heavy fermion properties of the Kondo Lattice model. Sci. Rep. 3, 2691; DOI:10.1038/srep02691 (2013).

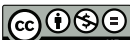

This work is licensed under a Creative Commons Attribution-

NonCommercial-NoDerivs 3.0 Unported license. To view a copy of this license, visit http://creativecommons.org/licenses/by-nc-nd/3.0 